\title{
Spark Ignition Engine Fuel-to-Air Ratio Control: An Adaptive Control Approach
}

\author{
Yildiray Yildiz*a, Anuradha M. Annaswamy ${ }^{\mathrm{a}}$, Diana Yanakiev ${ }^{\mathrm{b}}$, \\ Ilya Kolmanovsky ${ }^{\mathrm{b}}$ \\ ${ }^{a}$ Department of Mechanical Engineering, Massachusetts Institute of Technology, \\ Cambridge, MA, 02139 USA \\ ${ }^{b}$ Research and Innovation Center, Ford Motor Company, Dearborn, MI, 48121 USA
}

\section{Abstract}

This paper presents the control of Spark Ignition (SI) Internal Combustion (IC) engine Fuel-to-Air Ratio (FAR) using an adaptive control method of time-delay systems. The objective is to maintain the in-cylinder FAR at a prescribed set point, determined primarily by the state of the Three-Way Catalyst (TWC), so that the pollutants in the exhaust are removed with the highest efficiency. The FAR controller must also reject disturbances due to canister vapor purge and inaccuracies in air charge estimation and wallwetting (WW) compensation. Two adaptive controller designs are considered. The first design is based on feedforward adaptation while the second design is based on both feedback and feedforward adaptation incorporating the recently developed Adaptive Posicast Controller (APC). Both simulation and experimental results are presented demonstrating the performance improvement by employing the APC. Modifications and improvements to the

\footnotetext{
*Corresponding author. Tel.: +1 650604 4382; fax: +1 6506044036 .

Email addresses: yildiray.yildiz@nasa.gov (Yildiray Yildiz), aanna@mit.edu (Anuradha M. Annaswamy), dyanakie@ford.com (Diana Yanakiev), ikolmano@ford.com (Ilya Kolmanovsky) 
APC structure, which were developed during the course of experimentation to solve specific implementation problems, are also presented.

Key words: Internal combustion engines, Automotive control, Automotive emissions, Adaptive control, Delay compensation

\section{Introduction}

Triggered by the governmental regulations on emissions in 1960's and 1970's, the introduction of the microprocessor based control permitted the automotive manufacturers to design cleaner, more fuel efficient, better performing and more reliable powertrain systems. The associated control problems provide continuing challenges to control engineers as the requirements progressively become more stringent. Higher levels of performance and robustness are expected, while the calibration time and effort need to be reduced. Advances in control theory can be exploited to address these challenges. See (Guzzella and Onder, 2004) for an introduction to modeling and control of internal combustion engines.

The Fuel-to-Air Ratio (FAR) control is one of the most important control problems for conventional gasoline engines. The FAR control performance can strongly impact key vehicle attributes such as emissions, fuel economy and drivability. For instance, the FAR in engine cylinders must be controlled in such a way that the resulting exhaust gases can be efficiently converted by the Three-Way Catalyst (TWC). The TWC efficiency is about 98 percent when the fuel is matched to air charge in stoichiometric proportion and drops abruptly outside a narrow region as seen in Fig. 1 (Shelef and McCabe, 2000). The TWC can also compensate for the temporary FAR deviation from 
stoichiometry, by either storing excess oxygen or releasing oxygen to convert excess hydro-carbons (HC) and carbon monoxide (CO). Thus, for the TWC to operate efficiently, the stored oxygen level must be regulated so that a range to accommodate further release or storage during transient conditions is available (Guzzella and Onder, 2004). The oxygen storage level in the TWC may be inferred on the basis of the TWC model and a signal from a switching Heated Exhaust Gas Oxygen (HEGO) sensor located downstream of the TWC. In addition, the oxygen storage capacity of the TWC depends on the size and precious metal loading of the TWC. Therefore, if the FAR excursions and their durations are reduced with a well-performing controller, the storage capacity of TWC and its cost may be reduced as well.

A conventional FAR control system includes two nested controllers. The outer-loop controller generates a reference FAR (set-point) for the inner-loop controller based, for instance, on the deviation of the estimated TWC stored oxygen state. The inner-loop controller maintains the FAR upstream of the TWC at this set-point by using the measurements of the feedgas FAR with a linear Universal Exhaust Gas Oxygen (UEGO) sensor to appropriately correct engine fueling rate. Small amplitude low frequency periodic modulation may be superimposed over the set-point to further improve catalyst efficiency. The HEGO sensor downstream of the TWC is also used to improve robustness to UEGO sensor drifts, changes to fuel type, and for diagnostics.

The inner loop controller consists of a feedforward component which is fast but may not be always accurate, and a feedback component which is slower but eliminates the steady-state error (Guzzella and Onder, 2004). The feedforward component consists of estimation of the air and fuel path dynam- 


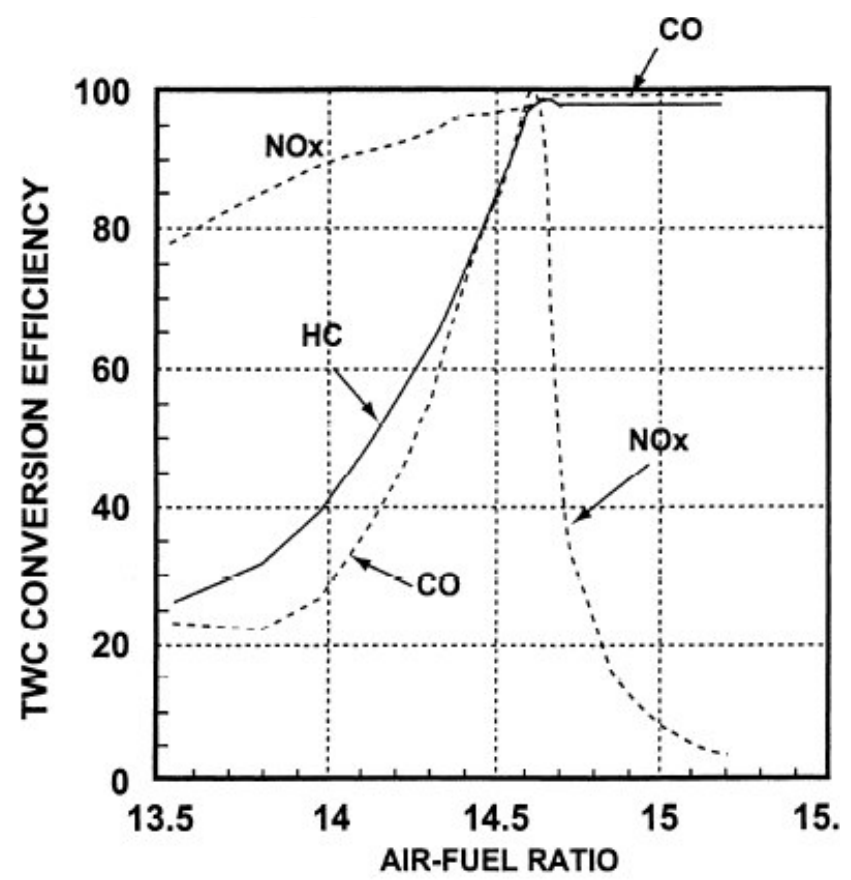

Figure 1: TWC efficiency vs. air-to-fuel ratio. 
ics combined with appropriate compensations. These air and fuel dynamics correspond, mainly, to the intake manifold lag that affects the air charge, and the wall-wetting (WW) that determines the amount of fuel inducted into the cylinder for each fuel injection event during transient operation.

The FAR control problem has been extensively investigated over many years. In terms of advanced approaches, the use of nonlinear feedforward controllers (Guzzella, 1995), adaptive controllers (Ault et al., 1994), (Turin and Geering, 1995), (Jones et al., 1995), (Rupp et al., 2008), (Rupp, 2009), feedback linearization (Guzzella et al., 1997), observer based controllers (Chang et al., 1995), (Powell et al., 1998), (Choi and Hedrick, 1998), sliding mode controllers (Won et al., 1998), (Pieper and Mehrotra, 1999), (Souder and Hedrick, 2004), linear quadratic regulators (Ohata et al., 1995), (Onder and Geering, 1993), $H_{\infty}$ controllers (Vigild et al., 1999), (Mianzo et al., 2001), Smith Predictors (Nakagawa et al., 2002), neural network controllers (Zhai and $\mathrm{Yu}$, in press) and model predictive controllers (Muske and Jones, 2006) can be mentioned. The use of an electronic throttle as an additional control actuator (Chang et al., 1993) or secondary/port throttles (Stefanopoulou et al., 1994) has been also explored. Apart from stoichiometric FAR controllers, reference (Zhang et al., 2007) considers control of FAR in a lean burn engine using linear parameter-varying controllers. In addition to these control research, (Tunestal and Hedrick, 2003) presents an interesting example of estimating the FAR in the cylinders without using the oxygen sensor and thus reducing the time delay in the system, and (Arsie et al., 2003) presents a research result on estimating the fuel-film dynamics. The motivation for these and related studies has been to achieve improved performance 
and robustness of the FAR control thereby enabling emission, fuel economy and drivability improvements.

Main challenges in the design of the FAR controller include variable time delay, uncertain plant behavior and disturbances. The time delay in the system comprises two basic components (Zhang et al., 2007): the time it takes from the fuel injection calculation to exhaust gas exiting the cylinders and the time it takes for the exhaust gases to reach the UEGO sensor location. The time delay in the system is a key factor limiting the bandwidth of the FAR feedback loop. The plant uncertainties are the result of inaccuracies in the air charge estimation and in the WW compensation, as well as changes in the UEGO sensor due to aging. When the carbon canister, which stores the fuel vapor generated in the fuel tank, is purged, the fuel content in the purge flow into the intake manifold is also uncertain and creates disturbance to the FAR control loop.

Therefore, a control approach which can handle both uncertainties and large time-delays, and that can achieve a high performance is of interest. Literature, given above, about classical and advanced control applications to the FAR control problem proves the success of an automatic, model based control approach, and this work built upon these results by eliminating the need of a precise engine model for classical or optimization based algorithms and by eliminating the conservatism introduced by the robust control approaches. This is achieved by using the Adaptive Posicast Controller (APC) (Niculescu and Annaswamy, 2003), (Yildiz et al., 2009a), which is an adaptive controller for time delay systems. Successful adaptive control approaches are presented also in references (Ault et al., 1994), (Turin and Geering, 1995), (Jones et al., 
1995), (Rupp et al., 2008) and (Rupp, 2009), but the approach presented in this paper is different from them: In (Ault et al., 1994) and (Jones et al., 1995), a nonlinear least squares parameter identification method is used to identify the plant parameter values online and then use these values in the controller. For the convergence of these parameters, the condition of persistent excitation is needed. In addition, this online parameter identification may require extra computational power. In both of the references, the controllers are applied to a single cylinder laboratory engine. In (Turin and Geering, 1995), again a similar approach is taken where an extended Kalman Filter is used to identify the plant parameter values online. Similarly, in (Rupp et al., 2008), the authors use a step by step experimental procedure to identify the sensor time constant, during the time of operation, where a rich input excitation is needed for parameter convergence. In (Rupp, 2009), the adaptive internal model controller is successfully implemented with the assumption that the fuel path is known and only the oxygen sensor dynamics is uncertain. On the other hand, the approach presented in this paper is based on direct adaptation where an online parameter identification scheme is not used and uncertainties are not confined to oxygen sensor parameters only but are allowed to appear elsewhere in the overall plant dynamics. In addition, APC is applied to a Lincoln Navigator test vehicle with 8 cylinders, which makes the control task much harder due to cylinder to cylinder variations. Finally, in this work, not only the APC results are presented but also a comparison with the existing control design in the test vehicle and with a gain scheduled Smith Predictor are provided.

The Adaptive Posicast Control (APC) is a recently developed control 
design approach that is especially suited for plants with large time-delays (Niculescu and Annaswamy, 2003), (Yildiz et al., 2009a) and parametric uncertainties. The APC can be described as an adaptive controller that combines explicit delay compensation, using the classical Smith Predictor (Smith, 1959) and finite spectrum assignment (Manitius and Olbrot, 1979), and adaptation (Ichikawa, 1985), (Ortega and Lozano, 1988). Due to such a unique combination, the APC effectively deals with both uncertainties and large time-delays both of which are dominant features of the FAR control problem. Previously, the authors explained preliminary implementation results of this controller to idle speed control and FAR control problems in conference papers (Yildiz et al., 2007), (Yildiz et al., 2008a) and (Yildiz et al., 2008b). This paper expands on those results with further theoretical improvements, new experimental results and more detailed explanations of the experimental issues.

To fit the specific needs of the FAR application, APC design has been extended with additional features: First, an adaptive feedforward term is added which is crucial for disturbance rejection. Second, procedures are developed for the controller parameter initialization and the adaptation rate selection to reduce the calibration time and effort. Third, an algorithm to take care of the variable delay is introduced. Fourth, an anti-windup logic is used to prevent the winding up the integrators used for parameter adaptation. Finally, a robustifying scheme is used to prevent the drift of the adaptive parameters. The main contribution of this work is the demonstration of the potential of this adaptive controller to improve the performance and to reduce the time and effort required for the controller calibration. This is achieved 
by the help of modifications and improvements that are listed above.

The experimental results obtained using a Lincoln Navigator test vehicle provided by Ford Motor Company, Dearborn, USA, demonstrate the capability of the controller to improve performance and decrease the calibration time and effort.

Adaptive Posicast FAR control approach represents a step towards a fully self-calibrating FAR controller because it reduces reliance on feedforward characterization and because the controller gains are automatically tuned online.

For comparison with the APC, in this paper a feedforward adaptive controller is also developed that attempts to minimize the impact of the purge fuel disturbance. This controller is also compared with the baseline controller using simulations and in-vehicle experiments.

While the control approach is adaptive, its development both benefits from and depends on the structural properties of the underlying plant model. This plant model for FAR ratio control is briefly discussed next, while the reader is referred to (Guzzella and Onder, 2004) for a more extended treatment of the underlying modeling techniques.

\section{Plant Model}

A block diagram representation of the plant, from fuel injection to the universal exhaust gas oxygen (UEGO) sensor measurement, together with the TWC is shown in Fig. 2, where " $A$ " stands for the air charge that is calculated based on the driver torque command. The fuel inducted into the engine cylinders is viewed as the sum of the output the WW dynamics block 


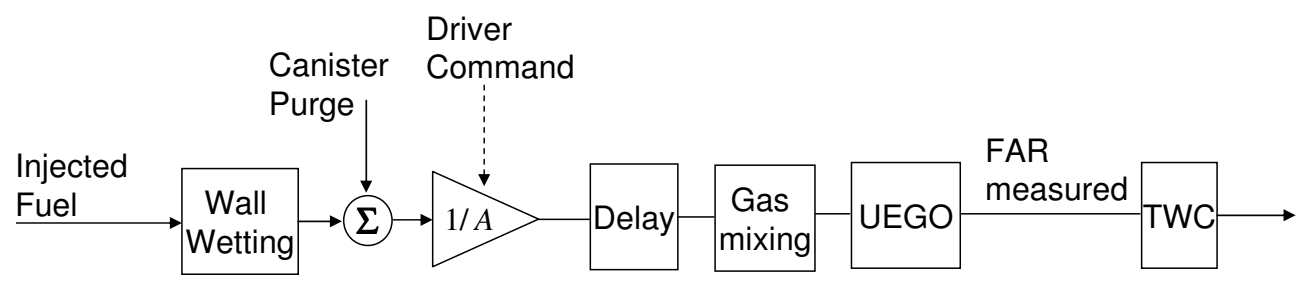

Figure 2: Plant block diagram representation.

and the canister purge, while the fuel injected by the injectors is an input to the WW block. The multiplication by the gain in the "1/A" block gives the FAR of the mixture in the engine cylinders (The control of FAR is considered as opposed to air-to-fuel ratio (AFR) since it scales linearly with fuel) and the delay block represents the combined effect of time delays in the system. The largest contributors to that delay are the time from the fuel injection to exhaust gas formation and the time needed for the exhaust gases to reach the UEGO sensor location. Finally, the exhaust gases undergo mixing, FAR is measured by the UEGO sensor and then the mixture passes through the TWC to get stripped from its pollutants.

In the model used, the input is the mass flow rate of fuel injected by the injectors and the output is the equivalence ratio, which is the fuel to air ratio normalized by its stoichiometric value, measured by the UEGO sensor in the exhaust. As explained above, there are mainly four components of the FAR dynamics which are wall-wetting dynamics, fuel-air mixture formation, mixture propagation to the UEGO sensor location and finally UEGO sensor dynamics. Below, the modeling aspects for each component together with their transfer functions are explained. 


\subsection{Wall-Wetting (WW) Dynamics}

After the fuel is injected by the injectors, some of the fuel immediately evaporates and is inducted into the engine cylinders, while the rest replenishes a liquid fuel puddle, which forms on the walls of the intake ports and on the intake valves. A fraction of the fuel evaporates from the liquid puddle and is also inducted into the engine cylinders. A WW dynamics model represents this phenomenon with the following transfer function.

$$
\frac{F_{\mathrm{c}}(s)}{F_{\mathrm{i}}(s)}=\frac{1+(1-X) \tau_{\mathrm{vs}}}{\tau_{\mathrm{v}} s+1}
$$

where $F_{\mathrm{c}}, F_{\mathrm{i}}, X$ and $\tau_{\mathrm{v}}$ represent the fuel entering the cylinders, injected fuel, the fraction of the fuel contributing to the fuel puddle and the puddle evaporation time constant, respectively.

\subsection{FAR Formation and Propagation to the UEGO Sensor}

The vaporized fuel mixes with the air and forms the fuel-air mixture. This process may be modeled as a division of the fuel mass by the air mass, $A(t)$, entering the cylinder. Starting from the opening of the intake valve, it takes approximately one engine cycle, i.e., 2 crankshaft revolutions, until

the exhaust gases fully exit the cylinder. This delay is called the cycle delay, $\tau_{\mathrm{c}}$, and can be approximated as $\tau_{\mathrm{c}}=120 / N$, where $N$ is the engine speed in revolutions-per-minute.

After the exhaust gases exit the cylinder, they mix with the previously existing exhaust gases and travel through the exhaust manifold until they reach the UEGO sensor location. Also, in a multi-cylinder engine, the exhaust gases coming from the individual cylinders enter the exhaust manifold 
at different times. All these effects can be modeled by a pure delay element in series with a first order lag as

$$
\frac{\Phi_{\mathrm{bm}}(s)}{\Phi_{\mathrm{eng}}(s)}=\frac{1}{\tau_{\mathrm{gm}} s+1} e^{-\tau_{\mathrm{tr}}}
$$

where, $\Phi_{\mathrm{bm}}, \Phi_{\mathrm{eng}}, \tau_{\mathrm{gm}}$ and $\tau_{\mathrm{tr}}$ represent the equivalence ratio, which is fuelto-air ratio divided by stoichiometric (desired) fuel-to-air ratio, just before the measurement, equivalence ratio right after the engine exit, gas mixing time constant and transport delay, respectively.

\subsection{Sensor Dynamics}

Sensor dynamics can be modeled by a first order lag as

$$
\frac{\Phi_{\mathrm{m}}(s)}{\Phi_{\mathrm{bm}}(s)}=\frac{1}{\tau_{\mathrm{s}} s+1}
$$

where $\Phi_{\mathrm{m}}(s)$ and $\tau_{\mathrm{s}}$ represent the measured equivalence ratio and sensor time constant, respectively.

\subsection{Reduced Order Model}

When all the individual elements of FAR dynamics described in eqns. (1)(3) are combined, a third order transfer function in series with a pure delay is obtained. To simplify the controller design, a first order lag in series with a pure time delay is used as a reduced order plant model, where the input and the output are the deviations in the commanded in-cylinder equivalence ratio and the measured equivalence ratio.

$$
G(s)=\frac{1}{\tau_{\mathrm{m}} s+1} e^{-\tau s}
$$


Accurate WW compensation (effectively, the feedforward inversion of (1)) helps to render this approximation more valid. Using relay feedback identification method for time delay systems (Majhi and Atherton, 2000) the coefficients of this model at around the speed of $700 \mathrm{rpm}$ and in warm conditions are found to be 0.4 and 0.45 for $\tau_{\mathrm{m}}$ and $\tau$, respectively. Note that $\tau$ consists of the cycle delay $\tau_{\mathrm{c}}$ and the transport delay $\tau_{\operatorname{tr}}$ and it also accounts for the UEGO sensor time delay and the computational delay in the engine control unit.

\section{Controller Design}

The structure of the closed loop system used in the test vehicle is presented in Fig. 3. The figure shows the inner and the outer control loops. The outer loop determines the desired FAR, $(F / A)_{\mathrm{d}}$, depending on the state of the TWC, measured by the HEGO sensor. $(F / A)_{\mathrm{d}}$ becomes the reference for the inner loop controller or the feedback controller, which is referred to as "Controller". The air estimate, referred to as $\hat{A}$, depends on the driver torque

request. The multiplication of $(F / A)_{\mathrm{d}}$ with $\hat{A}$ is referred to as the "base fuel", $F_{\mathrm{b}}$, which is an estimate of the desired fuel. The feedback controller corrects this estimate using the UEGO sensor measurement of the FAR upstream of the TWC. Note that the feedback controller applies a multiplicative correction as opposed to additive correction, although the latter is more typical in controls literature. The advantage of the multiplicative feedback over additive feedback is that the feedback fuel quantity scales proportionally to the value of the base fuel thereby providing better ability to compensate in transients when changes in vehicle operating point occur as this multiplicative 


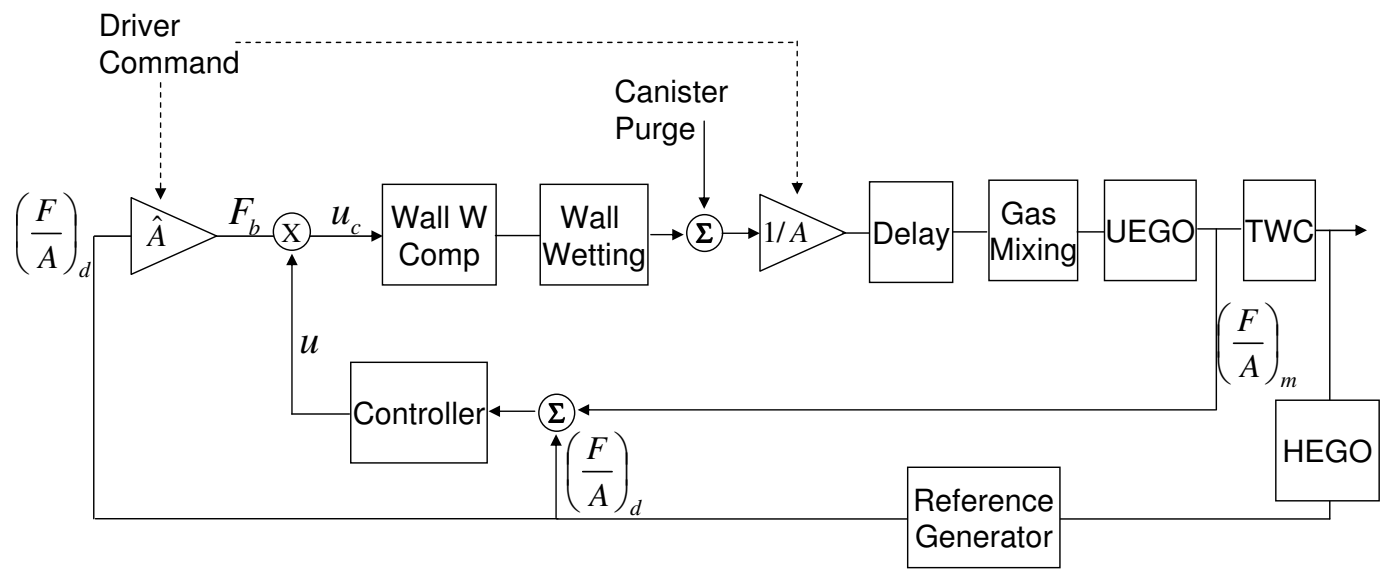

Figure 3: Overall closed loop controller structure.

transformation maintains the dc gain of the Plant assumed for fuel-to-air ratio feedback design constant across the vehicle operating range. In addition to the feedback controller there is a WW compensation algorithm in the system which estimates the WW dynamics and use the inverse dynamics to cancel it.

What is interested in is the feedback controller, for which two different adaptive controllers are designed with different complexity. Before explaining these designs, the baseline controller is explained first, which is the existing feedback controller in the vehicle.

\subsection{Baseline Controller}

The baseline controller in the vehicle is essentially a gain-scheduled Proportional - plus - Integral (PI) controller. In the actual vehicle implementation, a first-order filter in series with PI controller and relay logic are used. Note that before the feedback control input is multiplied by the base fuel $F_{\mathrm{b}}$, 
it is shifted by 1 . So the resulting control input for the baseline controller can be given as

$$
u_{\mathrm{c}}=(1+u) F_{\mathrm{b}},
$$

where $u_{\mathrm{c}}$ is the total control signal without the WW compensation and $u$ is the output of the feedback controller. This structure has the advantage of a feedback control input $u$ that has relatively small values, since it's bias, 1 , is already causing the control signal $u_{\mathrm{c}}$ to be equal to the required base fuel $F_{\mathrm{b}}$.

Note that, to maintain stability in the presence of delay, the gains of the PI controller cannot be made very aggressive. Moreover, due to the delay in the system, the overshoot in the response is difficult to avoid using this feedforward-feedback combination.

\subsection{Adaptive Feedforward Controller (AFFC)}

The system diagram with the Adaptive Feedforward Controller (AFFC) is shown in Fig. 4. This is a simple model reference adaptive controller, where it is assumed that the only uncertainty occurs in the control input gain. Instead of the feedback path in Fig. 3, a gain multiplier on the $(F / A)_{\mathrm{d}}$ is adapted. Note that the outer loop is not shown in the figure. The motivation for AFFC is to compensate for errors in the base fuel calculation due to, for example, injector uncertainties or "lost-fuel" effects present at cold engine conditions. Assuming that the desired FAR is in general constant and equal to stoichiometric FAR, it can be shown that this controller can also reject constant disturbances.

To derive the adaptation law, consider the following reduced order plant 


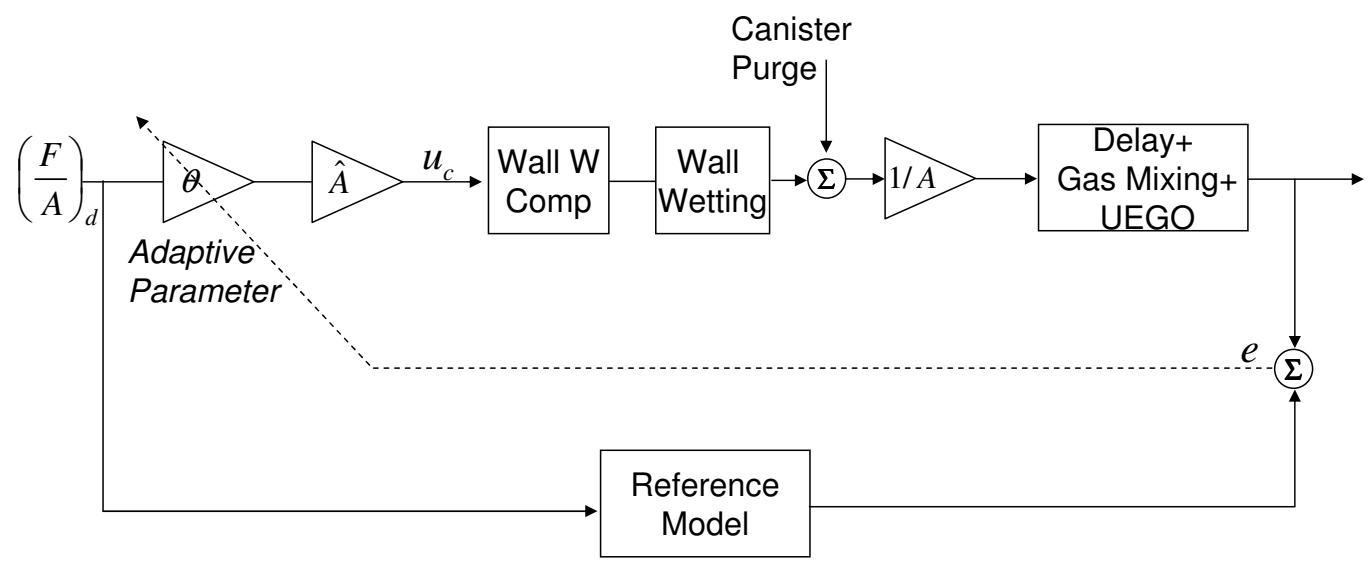

Figure 4: Inner-loop structure with AFFC.

model represented in state space form with a constant disturbance

$$
\dot{x}_{\mathrm{p}}=a x_{\mathrm{p}}+b(u(t-\tau)+d)
$$

where, $x_{\mathrm{p}}$ represents the measured FAR, $a$ and $b$ are known and unknown constants respectively and $d$ is a constant, unknown disturbance. Since the plant is stable, $a$ is negative and since $b$ represents the gain of the injectors, it is positive. Note that since reduced order dynamics is first order, (6) is a scalar differential equation.

Consider a reference model

$$
\dot{x}_{\mathrm{m}}=a x_{\mathrm{m}}+b_{\mathrm{m}} r(t-\tau)
$$

where $r$ is the desired FAR or $(F / A)_{\mathrm{d}}$. Assuming that the reference FAR is constant so that $r=r_{0}$, the control input is chosen as

$$
u=\theta r_{0}=\left(\theta^{*}+\tilde{\theta}(t)\right) r_{0}
$$


where $\theta^{*}=b_{\mathrm{m}} / b-d / r$ is the ideal controller parameter and $\tilde{\theta}$ is the deviation of $\theta$ from $\theta^{*}$. By using a Lyapunov function candidate $V=\left(e^{2}+b \tilde{\theta}^{2}\right) / 2$, it can be shown that $\tilde{\theta}(t)$ is bounded and $\lim _{t \rightarrow \infty} e(t)=0$, if the following update law is used

$$
\dot{\tilde{\theta}}=\dot{\theta}=-\gamma e r
$$

where $\gamma$ is the adaptation rate and $e=(F / A)_{\mathrm{m}}-(F / A)_{\mathrm{rm}}$. Here $(F / A)_{\mathrm{m}}$ and $(F / A)_{\text {rm }}$ represents the measured FAR and the output of the reference model.

One of the advantages of AFFC is providing a more damped response compared to the baseline controller when the goal is reference tracking. In addition AFFC is easier to tune since it has only one parameter. Note that an adaptation law (9) can be enhanced with a dead-band or a sigma-modification and with a projection algorithm.

\subsection{Adaptive Posicast Controller (APC)}

As discussed above, AFFC can handle the uncertainties in the controller gain. However, in reality there are more uncertainties in the system resulting from, for example, the actuator aging or simply from the operating point changes such as varying temperatures, speeds and loads. All these effects can change the system dominant pole. Therefore, a controller is needed that can also adapt to these uncertainties, which makes the APC a good candidate.

APC is a model reference adaptive controller for systems with known input delay. Below, the main idea behind the APC is summarized. The the reader is referred to (Yildiz et al., 2009a) for additional details. Consider a 
linearized plant with input-output description given as

$$
y(t)=W_{\mathrm{p}}(s) u(t-\tau), \quad W_{\mathrm{p}}(s)=\frac{k_{\mathrm{p}} Z_{\mathrm{p}}(s)}{R_{\mathrm{p}}(s)}
$$

where $y$ is the measured plant output, $u$ is the control input, and $W_{\mathrm{p}}(s)$ is the delay-free part of the plant transfer function. $R_{\mathrm{p}}(s)$ is the $n^{\text {th }}$ order denominator polynomial, not necessarily stable and the numerator polynomial, $Z_{\mathrm{p}}(s)$ has only minimum phase zeros. The relative degree, $n^{*}$, which is equal to the order of the denominator minus the order of the numerator, is assumed to be smaller or equal to two. It is also assumed that the delay and the sign of the high frequency gain $k_{\mathrm{p}}$ are known, but otherwise $W_{\mathrm{p}}(s)$ may be unknown. Suppose that the reference model, reflecting desired response characteristics, is given as

$$
y_{\mathrm{m}}(t)=W_{\mathrm{m}}(s) r(t-\tau), \quad W_{\mathrm{m}}(s)=\frac{k_{\mathrm{m}}}{R_{\mathrm{m}}(s)}
$$

where $R_{\mathrm{m}}(s)$ is a stable polynomial with degree $n^{*}, k_{\mathrm{m}}$ is the high frequency gain and $r$ is the desired reference input.

Consider the following state space representation of the plant dynamics (10), together with two "signal generators" formed by a controllable pair $\Lambda, l$

$$
\begin{aligned}
& \dot{x}_{\mathrm{p}}(t)=A_{\mathrm{p}} x_{\mathrm{p}}(t)+b_{\mathrm{p}} u(t-\tau), y(t)=h_{\mathrm{p}}^{T} x_{\mathrm{p}}(t) \\
& \dot{\omega}_{1}(t)=\Lambda \omega_{1}(t)+l u(t-\tau) \\
& \dot{\omega}_{2}(t)=\Lambda \omega_{2}(t)+l y(t)
\end{aligned}
$$

where, $\Lambda \in \Re^{n x n}$ and $l \in \Re^{n}$. It follows (Narendra and Annaswamy, 2005) that there exist $k^{*} \in \Re, \alpha_{1}^{* T}, \alpha_{2}^{* T} \in \Re^{n}, \lambda^{*}(\sigma):[-\tau, 0] \rightarrow \Re$ such that the control law

$$
u(t)=\alpha_{1}^{* T} \omega_{1}(t)+\alpha_{2}^{* T} \omega_{2}(t)+\int_{-\tau}^{0} \lambda^{*}(\sigma) u(t+\sigma) \mathrm{d} \sigma
$$




$$
+k^{*} r(t)
$$

satisfies the exact model matching condition.

$$
\frac{y(t)}{r(t)}=\frac{k_{\mathrm{m}}}{R_{\mathrm{m}}(s)} e^{-\tau s} .
$$

Now, the control of the plant (10) is considered when the transfer function $W_{\mathrm{p}}(s)$ has unknown coefficients and the time delay $\tau$ is known. Consider the following adaptive controller (Yildiz et al., 2009a):

$$
\begin{aligned}
u(t)= & \alpha_{1}(t)^{T} \omega_{1}(t)+\alpha_{2}(t)^{T} \omega_{2}(t)+\int_{-\tau}^{0} \lambda(t, \sigma) u(t+\sigma) \mathrm{d} \sigma \\
& +k(t) r(t), \\
\dot{\theta}(t)= & -\Gamma e_{1}(t) \Omega(t-\tau), \\
\frac{\partial \lambda(t, \sigma)}{\partial t}= & -\gamma_{\lambda}(\sigma) e_{1}(t) u(t+\sigma-\tau)
\end{aligned}
$$

where,

$$
\theta=\left[\begin{array}{c}
\alpha_{1} \\
\alpha_{2} \\
k
\end{array}\right], \quad \Omega=\left[\begin{array}{c}
\omega_{1} \\
\omega_{2} \\
r
\end{array}\right], \quad e_{1}=y-y_{\mathrm{m}},
$$

$\Gamma$ is a diagonal matrix, the entries of which represent the adaptation rate of the corresponding controller parameter and $\gamma_{\lambda}(\sigma)$ is the adaptation rate for the controller parameter $\lambda(t, \sigma)$. Defining the parameter errors as $\tilde{\theta}(t)=$ $\theta(t)-\theta^{*}, \tilde{\lambda}(t, \sigma)=\lambda(t, \sigma)-\lambda^{*}(\sigma)$, the control signal $u$ in (17) can be rewritten as

$$
\begin{aligned}
u(t)= & \alpha^{* T} \omega(t)+\int_{-\tau}^{0} \lambda^{*}(\sigma) u(t+\sigma) \mathrm{d} \sigma \\
& +k^{*} r(t) \\
& +\tilde{\alpha}(t)^{T} \omega(t)+\int_{-\tau}^{0} \tilde{\lambda}(t, \sigma) u(t+\sigma) \mathrm{d} \sigma \\
& +\tilde{k}(t) r(t)
\end{aligned}
$$


where $\alpha \triangleq\left[\begin{array}{ll}\alpha_{1} & \alpha_{2}\end{array}\right]$. It is shown in (Yildiz et al., 2009a) that the differential equations, (12), (13), (14) together with the control signal (19) describe the closed loop dynamics as

$$
\begin{aligned}
& \dot{X}_{\mathrm{p}}(t)=A_{\mathrm{m}} X_{\mathrm{p}}(t)+b_{\mathrm{m}}\left[\tilde{\alpha}^{T}(t-\tau) \omega(t-\tau)\right. \\
& \left.+\int_{-\tau}^{0} \tilde{\lambda}(t-\tau, \sigma) u(t-\tau+\sigma) \mathrm{d} \sigma+\tilde{k}(t-\tau) r(t-\tau)+k^{*} r(t-\tau)\right], \\
& y_{\mathrm{p}}(t)=h_{\mathrm{m}}^{T} X_{\mathrm{p}}(t)
\end{aligned}
$$

where, $X_{\mathrm{p}} \triangleq\left[\begin{array}{ccc}x_{\mathrm{p}}^{T} & \omega_{1}^{T} & \omega_{2}^{T}\end{array}\right]^{T}, h_{\mathrm{m}}^{T} \triangleq\left[\begin{array}{lll}h_{\mathrm{p}}^{T} & 0 & 0\end{array}\right], y_{\mathrm{p}}=y$ and $A_{\mathrm{m}}$ is a constant Hurwitz matrix. From the model matching condition, it is known that when the parameter errors are equal to zero, the closed loop transfer function is identical to that of the reference model. Therefore, the reference model can be described by the $(3 n)^{\text {th }}$ order differential equation

$$
\dot{X}_{\mathrm{m}}(t)=A_{\mathrm{m}} X_{\mathrm{m}}(t)+b_{\mathrm{m}} k^{*} r(t-\tau), y_{\mathrm{m}}(t)=h_{\mathrm{m}}^{T} X_{\mathrm{m}}(t)
$$

where,

$$
\begin{aligned}
& X_{\mathrm{m}}(t) \triangleq\left[\begin{array}{lll}
x_{\mathrm{p}}^{* T} & \omega_{1}^{* T} & \omega_{2}^{* T}
\end{array}\right]^{T}, \\
& h_{\mathrm{m}}^{T}\left(s I-A_{\mathrm{m}}\right)^{-1} b_{\mathrm{m}} k^{*}=\frac{k_{\mathrm{m}}}{R_{\mathrm{m}}(s)} .
\end{aligned}
$$

Note that $x_{\mathrm{p}}^{*}(t), \omega_{1}^{*}(t)$ and $\omega_{2}^{*}(t)$ can be considered as the signals in the reference model corresponding to $x_{\mathrm{p}}(t), \omega_{1}(t)$ and $\omega_{2}(t)$ in the closed loop system. Therefore, subtracting (21) from (20), an error equation for the overall system is obtained as

$$
\begin{aligned}
\dot{e}(t)= & A_{\mathrm{m}} e(t)+b_{\mathrm{m}}\left[\tilde{\alpha}^{T}(t-\tau) \omega(t-\tau)\right. \\
& +\int_{-\tau}^{0} \tilde{\lambda}(t-\tau, \sigma) u(t-\tau+\sigma) \mathrm{d} \sigma
\end{aligned}
$$




$$
\begin{aligned}
& +\tilde{k}(t-\tau) r(t-\tau)] \\
e_{1}(t)= & h_{\mathrm{m}}^{T} e(t)
\end{aligned}
$$

where $e(t)=X_{\mathrm{p}}-X_{\mathrm{m}}$ and $e_{1}(t)=y_{\mathrm{p}}(t)-y_{\mathrm{m}}(t)$. Equation (23) can be written in a more compact form as

$$
\begin{aligned}
\dot{e}(t)= & A_{\mathrm{m}} e(t)+b_{\mathrm{m}}\left[\tilde{\theta}^{T}(t-\tau) \Omega(t-\tau)\right. \\
& \left.+\int_{-\tau}^{0} \tilde{\lambda}(t-\tau, \sigma) u(t-\tau+\sigma) \mathrm{d} \sigma\right] \\
e_{1}(t)= & h_{\mathrm{m}}^{T} e(t) .
\end{aligned}
$$

Using the error model (24) and defining an appropriate Lyapunov Krasovskii functional, it can be shown (Yildiz et al., 2009a) that the plant (10), adaptive controller and the adaptive laws given in (17) have bounded solutions for all $t \geqslant t_{0}$ and $\lim _{t \rightarrow \infty} e_{1}(t) \rightarrow 0$.

\subsection{Implementation Enhancements}

In order to implement the Adaptive Posicast Controller specified by (13), (14) and (17), one has to address several issues which were not taken into account during the initial design but arise in the implementation. Below, these issues and how they are addressed are explained.

\subsubsection{Disturbance rejection}

Controller (17) is a model reference adaptive controller where the goal is to force the plant output follow the reference model output. In the design stage, the input disturbances are not explicitly taken into account. However, in the FAR control application, it can be shown that the controller is rejecting constant input disturbances. Indeed, the reference, FAR set-point, is 
constant for this application, which turns the feedforward term $k(t) r(t)$ into a pure integrator. Please see Appendix A for the proof of the disturbance rejection.

\subsubsection{Initialization and Adaptation Rate Selection}

Controller parameters are initialized by satisfying the model matching using a nominal plant model. For the nominal plant $700 \mathrm{rpm}$ is chosen as the engine speed at warm idling conditions. Idling can be considered as the worst case since the delay value achieves its maximum value. In this operating condition $\tau$ and $\tau_{\mathrm{m}}$ is found to be 0.4 and 0.45 respectively.

The adaptation gain $\bar{\Gamma}_{\mathrm{ii}}$ for a particular controller parameter $\bar{\theta}_{\mathrm{i}}$ is chosen using the following empirical rule

$$
\bar{\Gamma}_{\mathrm{ii}}=c \bar{\theta}_{\mathrm{i} 0}
$$

where $c$ is an adjustable gain and $\bar{\theta}_{\mathrm{i} 0}$ is the initial value of the corresponding controller parameter. Note that the same $c$ is used for all the parameters which makes the fine tuning procedure easy and fast. The rationale for this rule is to make all the controller parameters equally effective in the control law.

\subsubsection{Approximation of the finite integral term}

The finite integral term in the control signal $u$ given in (17) is implemented by using a set of point-wise delays (Manitius and Olbrot, 1979) as in the following:

$$
\int_{-\tau}^{0} \lambda(\sigma, t) u(t+\sigma) \mathrm{d} \sigma=\lambda_{1}(t) u(t-d t)+. .+\lambda_{m}(t) u(t-m d t)
$$


where $d t$ is the sampling interval and $m d t=\tau$. With this approximation, the adaptive laws given in (17) can be represented as

$$
\dot{\bar{\theta}}(t)=-\bar{\Gamma} e_{1}(t) \bar{\Omega}(t-\tau)
$$

where,

$$
\bar{\theta}=\left[\begin{array}{c}
\alpha_{1} \\
\alpha_{2} \\
\lambda_{1} \\
\vdots \\
\lambda_{m} \\
k
\end{array}\right], \quad \bar{\Omega}=\left[\begin{array}{c}
\omega_{1} \\
\omega_{2} \\
u(t-d t) \\
\vdots \\
u(t-m d t) \\
r
\end{array}\right],
$$

and $\bar{\Gamma}$ is the diagonal adaptation rate matrix.

In (Engelborghs et al., 2001) the limitations of this approximation have been pointed out together with an example of unstable behavior arising due to numerical integration. In the powertrain control problem considered here, both in the experiments and in the simulations, the values of coefficients $\lambda_{i}$ are in the order of $10^{-3}$ to $10^{-4}$, and for these values the danger of the instabilities due to numerical approximation does not arise. Please see Appendix $\mathrm{C}$ in (Yildiz et al., 2009b) for details.

\subsubsection{Handling Time-Varying Delay}

In the design of the APC, it assumed that the time delay in the system is known and constant. However, the time delay in the FAR control problem varies with the load and the speed of the engine. A logical way of handling this issue is gain-scheduling the controller, time delay being the gain-scheduling variable. The delay value shows itself in the equation (13) 
and in the adaptation laws given in (17), which are straightforward to gainschedule. Apart from these, the finite integral term in the control law given in (17) also needs the delay information to be computed. Note that an approximation is used for this term given in (26). Two different strategies are pursued to gain-schedule this approximation, which are given below:

a) Eliminating and Adding Terms:

The integral in (26) is approximated using time steps that are equal to the sampling interval, $T$, of the controller implementation ( $T=30 \mathrm{~ms}$ ). Therefore, the number of the terms, $m$, in this approximation can be given as $m=\tau / T$. A simple way to gain-schedule this approximation is to eliminate or add terms, depending on the value of the delay at the time of approximation. One can do this by storing the values of eliminated parameter $\lambda_{i}$ 's when the delay decreases and then using these stored values when the number of the terms increases again, due to a delay increase.

Although this logic seems intuitive, it has a drawback of rapid control signal changes that can cause undesired excursions in the FAR trace.

b) Freezing and Adding Terms:

As discussed above, when the delay value decreases, less parameters are needed to approximate the finite integral in (26) and thus the unnecessary terms are eliminated. This causes a sudden, undesired jump in the control signal. To prevent this jump, instead of eliminating the unnecessary terms, $\lambda_{\mathbf{i}}(t) u(t-i d t)$ 's, they are simply frozen and used back when the delay value increases. This strategy achieves two things: First, it still makes sure that only the necessary terms are being used and thus only the necessary parameter $\lambda_{\mathrm{i}}$ 's are being updated, while the rest of them are frozen. Second, by still 
keeping the frozen terms in the control signal, it leads a smooth transition from one delay approximation to another.

Note that in the case of a delay decrease and thus freezing of the unnecessary terms, the control signal carries the frozen terms as a constant bias. Below, it is shown that this does not adversely effect the stability of the closed loop system.

Assume that the delay value $\tau$ decreased to $\tau^{\prime}$ so that only $p=\tau^{\prime} / T$ terms are needed instead of $m$ terms to approximate the finite integral, where $p<m$. In this case, $m-p$ unnecessary terms are frozen. Assume that the sum of these frozen terms are equal to $D$. The resulting approximation is the following:

$$
\int_{-\tau^{\prime}}^{0} \lambda(\sigma, t) u(t+\sigma) \mathrm{d} \sigma=\lambda_{1}(t) u(t-d t)+. .+\lambda_{p}(t) u(t-p d t)+D .
$$

The state space description of the plant together with the controller given in (12) is now modified as

$$
\dot{x}_{\mathrm{p}}(t)=A_{\mathrm{p}} x_{\mathrm{p}}(t)+b_{\mathrm{p}}(u(t-\tau)+D), y(t)=h_{\mathrm{p}}^{T} x_{\mathrm{p}}(t) .
$$

Therefore, the same procedure explained in Appendix A can be used to show that the overall system stays stable and that the tracking error goes to zero.

\subsubsection{Anti-windup logic}

The fuel injector actuators, have their hard limits and the calculated control signal may sometimes exceed these limits, either from below or from above. Consequently, an add-on algorithm needs to be integrated with the controller that prevents the winding up of the integrators resulting from the adaptation laws in (17). 
Anti-windup logic is used where the main goal is to stop the adaptation if the control signal saturates and if the tracking error, $e_{1}=y_{\mathrm{m}}-y_{\mathrm{p}}$, is not favorable. Calling the control signal before the saturation block as $u$ and after the saturation as $u_{\text {sat }}$, the anti-windup algorithm can be expressed as in the following.

$$
\dot{\bar{\theta}}_{\mathrm{i}}(t)=\left\{\begin{array}{cl}
0 & \text { if } \quad u>u_{\text {sat }} \text { and } e_{1}<0 \\
& \text { or } \\
& u<u_{\text {sat }} \text { and } e_{1}>0 \\
-\bar{\Gamma}_{\mathrm{ii}} e_{1}(t) \bar{\Omega}_{\mathrm{i}}(t-\tau) & \text { otherwise }
\end{array}\right.
$$

The additional tracking error based condition for not suspending the adaptation during saturation improved the speed of the transient response as has been demonstrated in vehicle experiments.

There are more rigorous anti-windup methods that are specifically developed for adaptive controllers (Karason and Annaswamy, 1994). These methods are planned to be used in future research.

\subsubsection{Robustness}

The adaptive controller design presented in Section 3.3 portrayed an idealized situation. The delay free part of the plant dynamics, $W_{\mathrm{p}}(s)$, is assumed to be finite dimensional, linear and time invariant with unknown parameters. It is also assumed that the inputs and outputs to the plant can be measured exactly. However, in the real implementation, no plant is truly linear or finite dimensional. Plant parameters may vary with time and operating conditions, and measurements may be contaminated by noise. The plant model is almost always approximate. It is precisely in these cases that adaptive control 
is most needed (Narendra and Annaswamy, 2005).

Due to the above possible violations of the assumptions, the controller parameters may drift without converging to a bounded region. One of the remedies to this problem is using $\sigma$ - modification robustness scheme (Narendra and Annaswamy, 2005), which mainly adds a damping term to adaptation laws. The authors previously used this robustness scheme in idle speed control application (Yildiz et al., 2009b) which proved successful and therefore it is used again in FAR control application. Please see (Yildiz et al., 2009b) for the details.

\subsection{Final Design and Calibration}

Any controller design that is meant to be used in a mass-production application must be accessible and easy to use by the engineering staff who actually implements and supports the control strategy in production. This is particularly important given that the engineering stuff are not expected to be highly skilled in advanced control methods. Motivated by these facts, a step by step design procedure to obtain a transparent and streamlined design is given below. It is assumed that a linear plant model with uncertain parameters and a known time delay is available.

Step 1. Select $\Lambda$ and $l$ of the signal generators defined in (13) and (14). These signal generators act like state observers and it is suggested that the eigenvalues are selected much faster than the reference model pole. Note that the $\Lambda$ - $l$ pair must be controllable.

Step 2. Set the initial value of the controller parameters by satisfying the model matching using a nominal plant model. 
Step 3. Set the time constant of the reference model at least two times faster than that of the nominal plant time constant.

Step 4. Set the adaptation rate matrix $\Gamma$ according to the algorithm given in (25).

Step 5. Tune the parameter $c$ until the control requirements are satisfied. Note that increasing $c$ gives a tighter FAR control performance, however higher gains might cause undesired oscillations.

Apart from these five easy steps, the design must be integrated with the robustness scheme as discussed in Section 3.4.6.

Note that the controller needs only about $0.4 \mathrm{~KB}$ of memory for the data storage and requires 118 number of operations per computation cycle. This corresponds to less than $4 \cdot 10^{3}$ operations per second. For conventional ECU's the APC controller use around 0.04 percent of the total computational power and that is negligible. Please see the appendix in (Yildiz et al., 2009b) for the calculation of the memory requirements and computational complexity.

\section{Simulation and Experimental Results}

The simulation results in this section are obtained using $®$ Matlab and (R) Simulink, and the experimental results are obtained using a Lincoln Navigator test vehicle provided by Ford Motor Company. The vehicle has a 5.4 liter V-8 front engine with a multi-port fuel injection system. The engine has three valves per cylinder and can achieve $300 \mathrm{Hp}$ at $5000 \mathrm{rpm}$ and $495 \mathrm{Nm}$ at $3750 \mathrm{rpm}$. The air intake is controlled with an electronic throttle. 


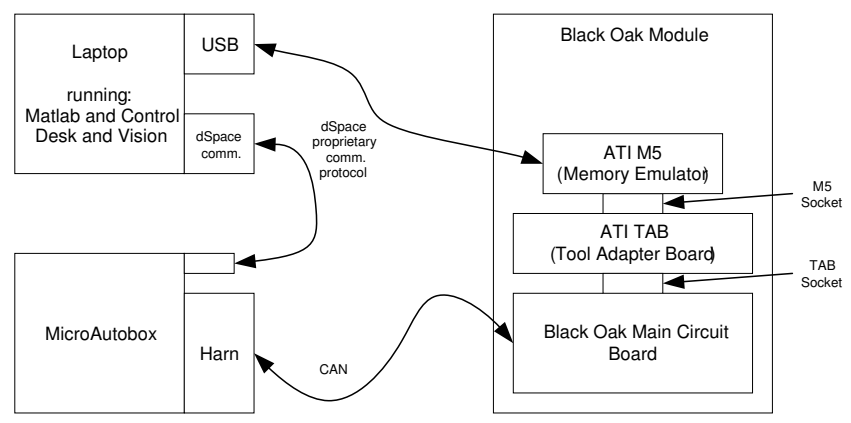

Figure 5: Rapid prototyping with MicroAutobox using CAN.

A dSPACE MicroAutoBox, communicating with the engine control unit (ECU) via CAN bus was used for real-time controller rapid prototyping. This system is used to implement the controller and monitor the performance. Figure 5 shows the hardware wiring. In the production environment, the engine is controlled by the ECU. The ECU normally also controls the other actuators of the engine, monitors the health of the engine and processes sensor inputs (van Nieuwstadt et al., 2000).

In the experimental setup, the FAR control commands coming from the ECU are overridden with the adaptive control signal by using the rapid prototyping system (see Figure 5). This system has the FAR as the measured input and calculates the fuel mass flow rate as the control input.

The existing closed loop control structure in the vehicle is presented in Fig. 3. The adaptive controller overwrites the "Controller" block, while the rest of the structure is retained as is. Thus, the presented results compare the performance of the existing feedback controller in the test vehicle with the adaptive controller. It was observed that the Adaptive Posicast Controller 
performed better when compared to the existing baseline controller, in all experiments.

\subsection{AFFC vs. Baseline Controller}

Figure 6 compares the tracking and purge disturbance rejection performance of the baseline controller and of the AFFC when WW dynamics are assumed to be perfectly compensated. $\Phi$ denotes the normalized FAR or the equivalence ratio (so that stoichiometric FAR of 0.0685 corresponds to $\Phi=1$. The upper plot shows the simulated response to a pulse train reference and the lower plot shows the response to a step purge disturbance introduced at time $t=30 \mathrm{sec}$ and removed at time $t=50 \mathrm{sec}$. It is assumed that the time delay is known to be 0.4 sec. While designing the AFFC, the UEGO dynamics are assumed to have nominal values but then the plant dynamics were chosen to have 20 percent deviations in high frequency gain and $\tau_{\mathrm{m}}$. The baseline controller is tuned to perform well for both tracking and disturbance rejection. As discussed before, the baseline controller cannot avoid overshoots due to the delay in the system, while the AFFC can track the reference comparatively better. On the other hand, the disturbance rejection capabilities are similar, since when the reference is constant, the AFFC is essentially an integral controller.

AFFC is also tested experimentally and compared with the existing baseline controller. At the test time, the calibration of WW compensation was not fully completed, which allowed to subject both controllers to challenging scenarios. Also, the time delay varied in the experiments as opposed to the cases simulated in Fig. 6. Figure 7 shows the results from a 4-minute drive test. Note that the air charge values have been scaled to show them in the 


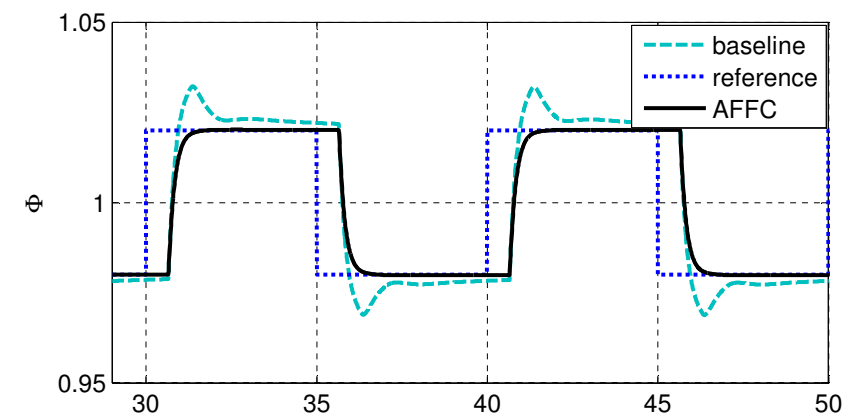

(a)

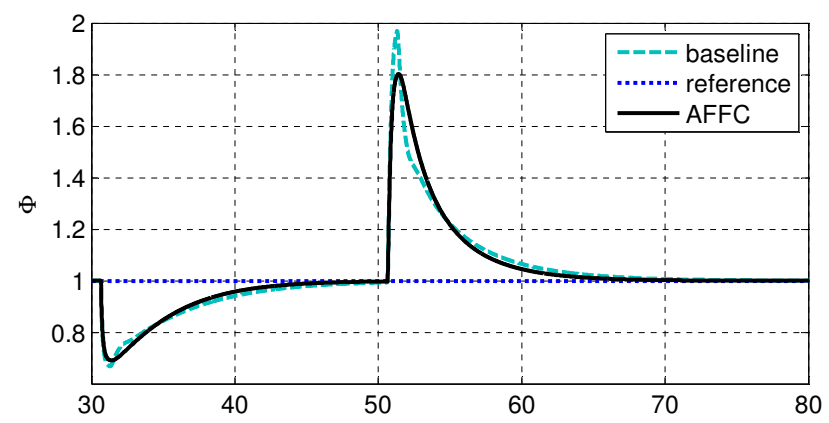

(b)

Figure 6: Comparison of baseline controller and AFFC. a) Response to a set-point change b) Response to purge disturbance. 


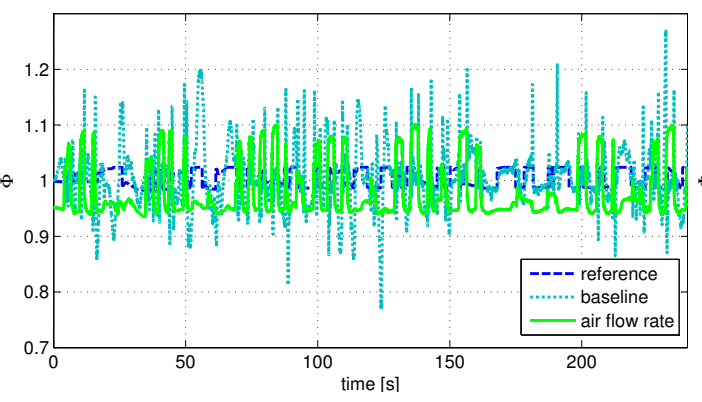

(a)

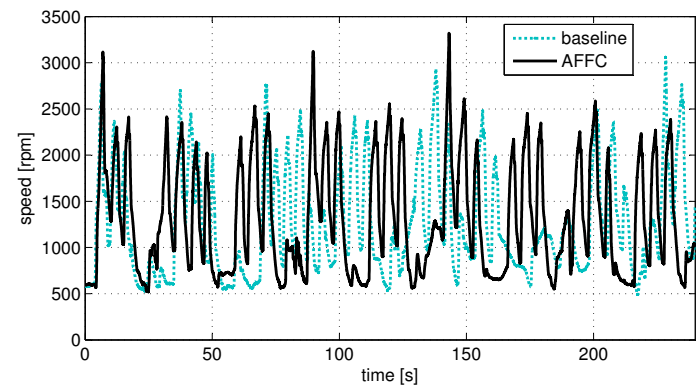

(c)

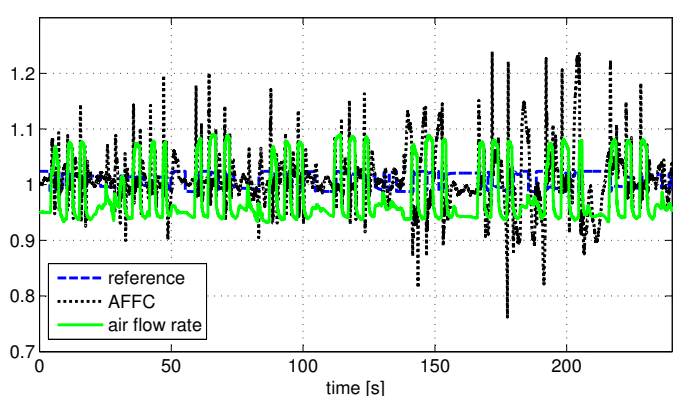

(b)

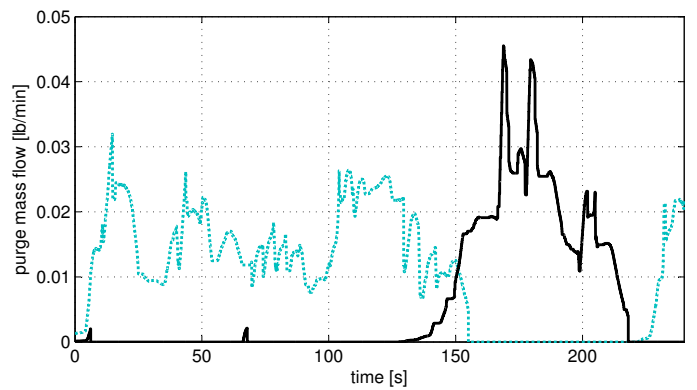

(d)

Figure 7: Baseline controller vs. AFFC a) $\Phi$ and air flow rate when baseline controller is active b) $\Phi$ and air flow rate when AFFC is active c) Engine speeds d) Purge fuel flow rates.

same plot with $\Phi$. The test was conducted in a relatively uncontrolled environment, e.g., without controlling the speed or load, as can be observed in Figs. 7a-c. The vehicle was accelerated and decelerated rather sharply and the purge flow was also not controlled, as shown in Fig. 7-d. The RMS error value of the deviations from the reference is calculated as 0.0052 and 0.0051 for the baseline controller and for the AFFC, respectively. Their performances are similar, consistent with the simulation results, as the dominant factors affecting the response are the purge and air disturbances, and not the reference tracking. 
Note that another important success measure (SM) for the FAR control loop is the error integral. Compared to RMS error, this metric better reflects how much of the TWC oxygen storage capacity is used to compensate for the deviations in the fuel-to-air ratio.

The integral error SM can be formulated as

$$
\mathrm{SM}=\frac{1}{k} \sum_{\mathrm{i}=1}^{k}\left|\int_{t_{i}}^{t_{i}+\Delta_{i}} e_{1}(\eta) \mathrm{d} \eta\right|
$$

where $t_{i}$ is the time instant of the $i$-th disturbance hit and $\Delta_{i}$ is the duration/settling time of the transient caused by the disturbance hit. This SM is used for the APC results in the following sections.

\subsection{APC vs. Baseline Controller}

\subsubsection{Purge Disturbance Rejection Tests}

The purpose of initial FAR control experiments was to compare the performances of the APC and the baseline controller, while emulating canister vapor purge disturbance rejection tests. These experiments were conducted with the test vehicle idling at different speeds. Since during idling the air flow rate does not change much, the WW dynamics did not play a major role in these experiments as much as it did for acceleration and deceleration experiments. The SM used is given in (32).

The test started with the engine speed at $700 \mathrm{rpm}$. At $300 \mathrm{sec}$, the engine speed increased to $1000 \mathrm{rpm}$ and at $600 \mathrm{sec}$ it decreased back to 700 rpm. Every $20 \mathrm{sec}$ the fuel injector gains were changed to emulate the purge disturbance. Overall, the performance of the APC, calculated using (32), was 70 percent better than the baseline controller during the test which lasted 15 


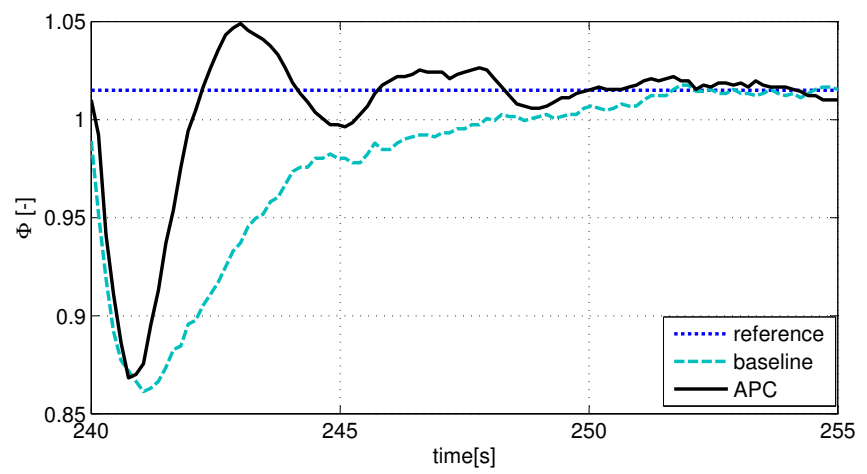

Figure 8: Comparison of baseline controller with APC for purge disturbance rejection at $700 \mathrm{rpm}$.

minutes. Figure 8 shows a time window from the test where the engine speed was $700 \mathrm{rpm}$. The APC performs considerably better, in terms of integral $\mathrm{SM}$, than the baseline controller as its features enable it to better account for the delay and achieve faster response.

Figure 9 shows how the equivalence ratio changes during the same test but now the engine speed is $1000 \mathrm{rpm}$. Again, the performance of the APC is better than that of the baseline controller.

\subsubsection{Acceleration and Deceleration Tests}

Figure 10 shows the equivalence ratio excursions during a test in which the vehicle accelerates and then decelerates. In this case, the delay varies with time during the test. The APC performs better overall than the baseline controller. During the lean excursion (equivalence ratio less than 1 during acceleration), the baseline controller appears to start the recovery from the undershoot slightly earlier than the APC. There are, however, differences in the air flow and the equivalence ratio set-point time of increase between APC 


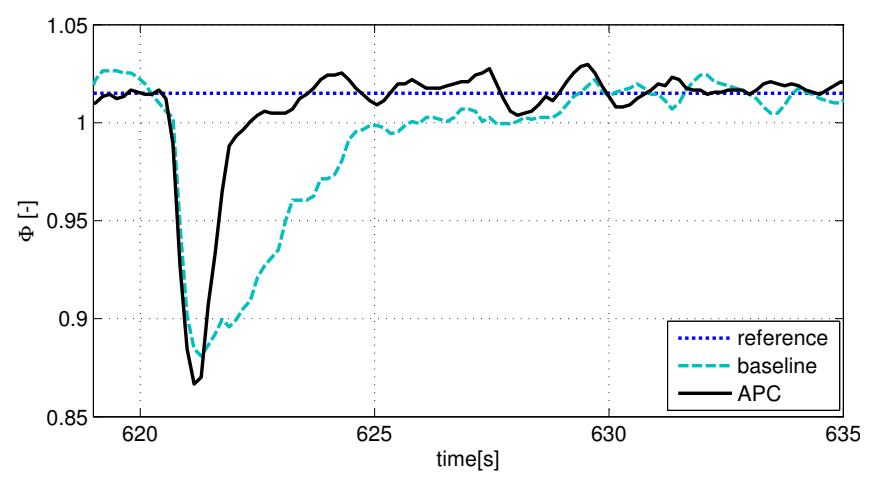

Figure 9: Comparison of baseline controller with APC for purge disturbance rejection at $1000 \mathrm{rpm}$, with $c=1$.

test and baseline controller test, further analysis of these suggests no real advantage for the baseline controller over APC in terms of start of recovery timing. Note that the equivalence ratio set-point is computed by a separate part of the engine control system in the vehicle.

For this experiment, the maximum value of the integrated difference between fuel-air equivalence ratio and its set point are compared during the full course of the experiment. This metric is better suited to assessing the difference between controllers for this experiment than (32) because if one acceleration-deceleration test is assumed to be a single event, the errors cancel each other if (32) is used, which can be observed in (10)-d. However, maximum value of the integral relates to how much of the oxygen storage capacity is used in the worst case during the course of the experiment. In terms of this metric, APC performs 43 percent better than the baseline controller.

All the above experiments were conducted with the fine tuning parameter $c$ equal to 1, which implies that no fine-tuning was done. In Fig. 11, an exper- 

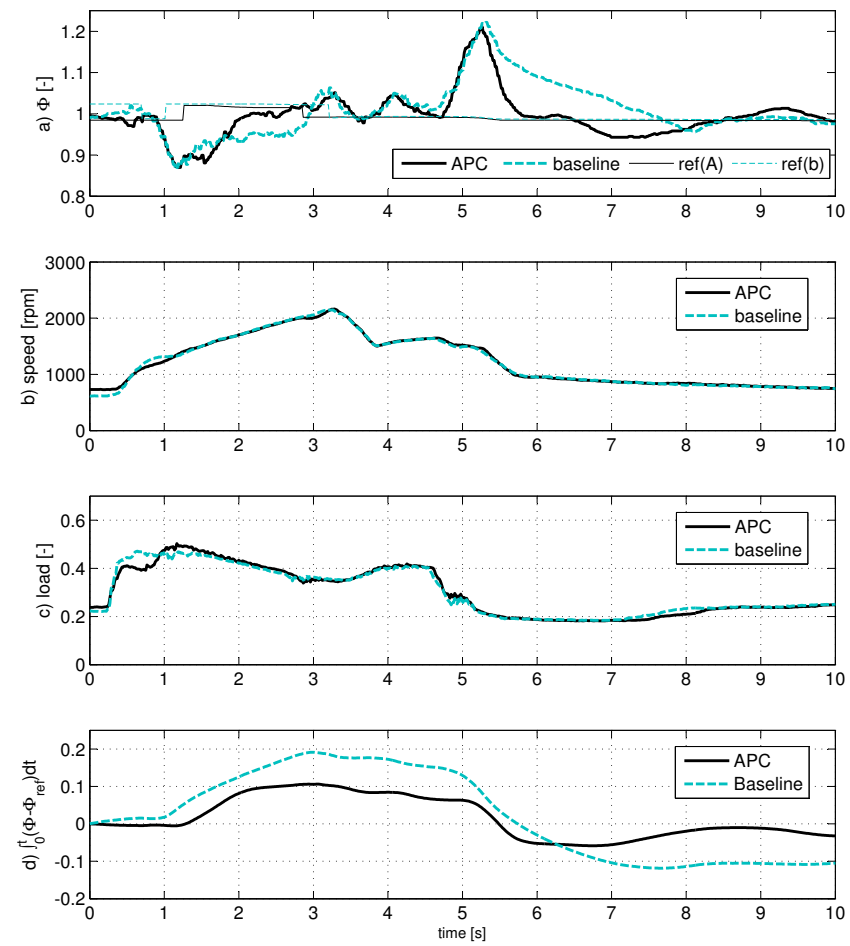

Figure 10: Time histories of a) $\Phi$, b) Engine speed c) Engine relative air flow, d) Tracking error integral, during vehicle acceleration and deceleration for APC vs. baseline controller, with $c=1$. 

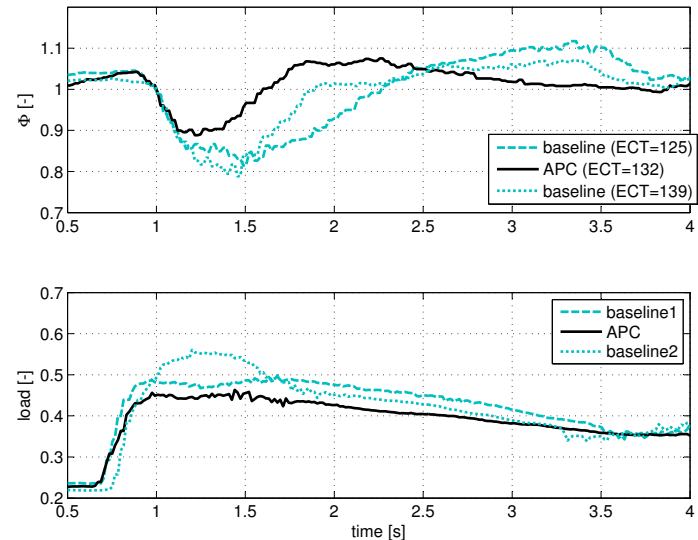

Figure 11: Comparison of baseline controller with APC during vehicle acceleration, with $c=1.5$.

imental result, which shows APC and the baseline controller performances during the vehicle acceleration, is presented with $c=1.5$. As expected, the APC outperforms the baseline controller to a greater extent compared to the previous cases, especially on lean excursions. Note however that the load (and hence the air charge) are less in the APC controller case in this experiment. Nevertheless, performance with the APC is considerably better than with the baseline controller, and cannot be attributed to the load difference between the controllers.

\subsection{APC vs. Gain-Scheduled Smith Predictor}

The performance of the APC was compared with a gain-scheduled Smith Predictor (SP). The SP was designed based on the plant models identified at different operating points (corresponding to different combinations of engine speeds and loads) using a relay feedback method. 


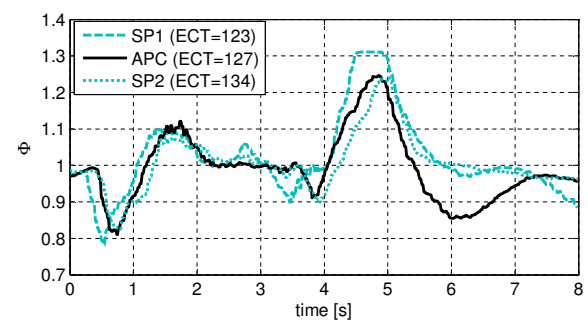

(a)

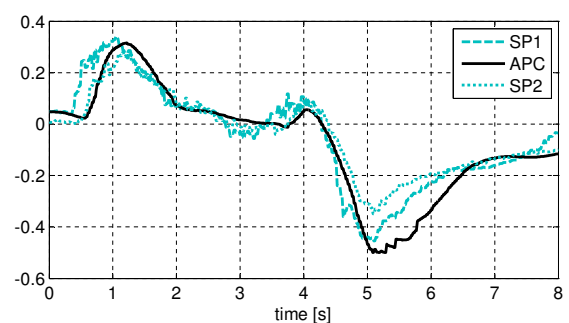

(c)

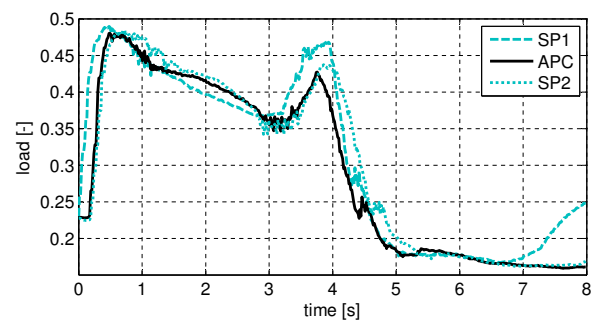

(b)

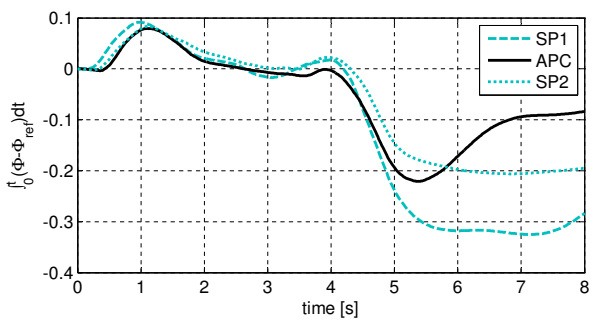

(d)

Figure 12: Time histories of a) $\Phi$ b) Engine relative air flow c) Feedback control input d) Tracking error integral, during vehicle acceleration and deceleration for gain-scheduled SP vs. APC, with $c=1.5$.

Figure 12 shows the results of an acceleration-deceleration test conducted using the test vehicle. The performances are very similar as can be seen in Fig. 12a and Fig. 12d, where the time evolutions of $\Phi$ and the error integral is presented. On the other hand, Fig. 12c shows that the control signal of the APC is smoother than that of the SP.

Figure 12 confirms that the adaptive controller is performing very well and similar in performance to the Smith Predictor. Note that the gain-scheduled SP can be seen as a perfect adaptive controller: While the APC adapts to operating point changes without the knowledge of the plant parameters, the gain-scheduled SP uses the knowledge of the changing plant parameters that 
need to be obtained offline by using an identification procedure for different operating points. The adaptive controller can, in addition, adjust better to situations when plant parameters change due to part-to-part variability or aging. For example, it is stated in (Rupp et al., 2008) that due to aging or harsh operating conditions, UEGO sensor time constant can easily increase by a factor of 10 to 20 . Also it is known that the Smith Predictor is sensitive to the delay estimation errors.

In Fig. 13, simulation results that compare SP with APC are presented. For this simulation, the time constant for the first order system model is selected as $50 \mathrm{~ms}$, which is reported in (Rupp et al., 2008) as the time constant of a state-of-the-art oxygen sensor. The nominal time delay is assumed to be 0.4 seconds. A step input disturbance is introduced to this plant at time $t=170$ seconds and the transients are plotted. The APC and the SP is tuned such that they perform similarly for these nominal plant parameter values, in the presence of the disturbance. Then, the sensor time constant is increased by a factor of 20 and the disturbance test is repeated. As seen in the figure, not only the performance of the SP gets worse than the adaptive controller, but the SP response also becomes oscillatory, which is a sign of getting closer to instability. An additional uncertainty in the system, like a delay identification error, may cause the system to become unstable easily. Indeed, when a delay uncertainty is introduced by increasing the nominal delay by 0.3 seconds, it is seen that the loop with the SP becomes almost marginally stable. This simulation result is presented in Fig 14 . 


\section{Summary}

In this paper, the fuel-to-air ratio (FAR) control problem in port-fuelinjection (PFI) spark-ignition (SI) engine is considered . Two controllers, an Adaptive FeedForward Controller (AFFC) and an Adaptive Posicast Controller (APC), have been developed and implemented in a test vehicle. The AFFC is a simple controller based on feedforward adaptation, while the APC is a more elaborate controller that uses adaptation in both feedforward and feedback paths and is based on a recently developed adaptive control method for time-delay systems. The AFFC has been shown in simulations and experiments to have better reference tracking and similar disturbance rejection capabilities when compared to the existing baseline controller. The APC has been shown in experiments to achieve faster recovery from disturbances and better performance during vehicle acceleration deceleration tests. These performance improvements were a result of various modifications and enhancements to the initial APC design, such as an algorithm to handle the variable time delay, a robustness scheme and parameter initialization and fine tuning methods. It has also been observed in vehicle experiments that implementing APC using an upper bound on the delay as a delay estimate assures robustness against delay variations.

In terms of applications of the APC, the FAR control problem is more challenging than the Idle Speed Control (ISC) problem, which the authors of this paper have treated in (Yildiz et al., 2007) and (Yildiz et al., 2009b), due to a larger and variable time delay and different character of disturbances and uncertainties. The experimental results reported here demonstrate that the APC is effective for the FAR control problem as well. 


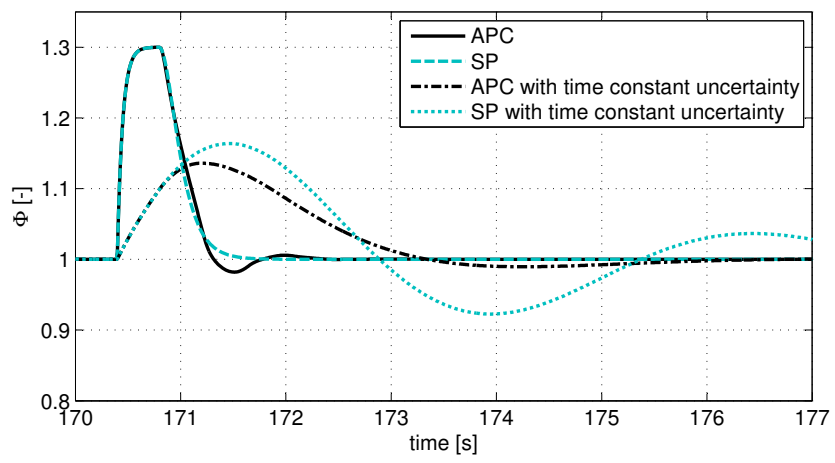

Figure 13: Comparison of SP and APC for input step disturbance rejection in the presence of sensor time constant uncertainty.

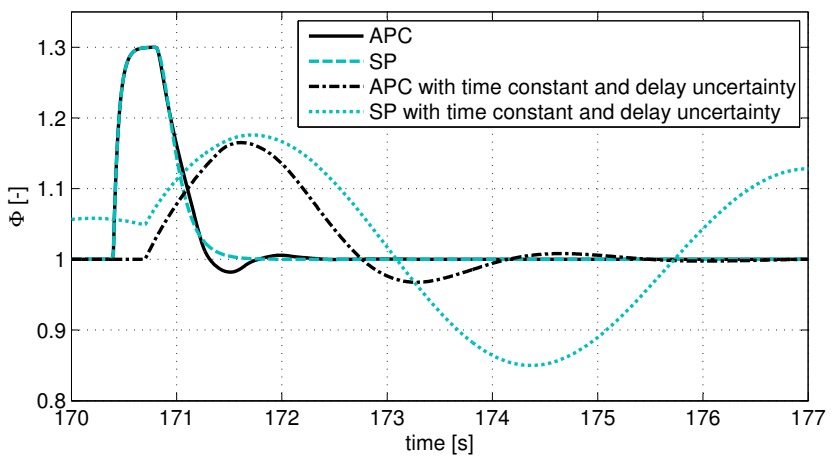

Figure 14: Comparison of SP and APC for input step disturbance rejection in the presence of sensor time constant and delay uncertainty. 


\section{A. Disturbance Rejection Proof}

When there is a constant disturbance $d \in \Re$ present in the system, the state space description of the plant (12) is modified as

$$
\dot{x}_{\mathrm{p}}(t)=A_{\mathrm{p}} x_{\mathrm{p}}(t)+b_{\mathrm{p}}(u(t-\tau)+d), y(t)=h_{\mathrm{p}}^{T} x_{\mathrm{p}}(t)
$$

This in turn modifies the error equation (23) as

$$
\begin{aligned}
\dot{e}(t)= & A_{\mathrm{m}} e(t)+b_{\mathrm{m}}\left[\tilde{\alpha}^{T}(t-\tau) \omega(t-\tau)\right. \\
& +\int_{-\tau}^{0} \tilde{\lambda}(t-\tau, \sigma) u(t-\tau+\sigma) \mathrm{d} \sigma \\
& +\tilde{k} r(t-\tau)+d] \\
e_{1}(t)= & h_{\mathrm{m}}^{T} e(t) .
\end{aligned}
$$

Note that in FAR control application, the FAR reference, $r_{0} \in \Re$, is constant and therefore $r(t-\tau)=r_{0}$ in (34). By defining a new variable $\tilde{k}^{\prime}$ as

$$
\tilde{k}^{\prime}=\tilde{k}+\frac{d}{r_{0}},
$$

equation (34) can be reduced to

$$
\begin{aligned}
\dot{e}(t)= & A_{\mathrm{m}} e(t)+b_{\mathrm{m}}\left[\tilde{\alpha}^{T}(t-\tau) \omega(t-\tau)\right. \\
& +\int_{-\tau}^{0} \tilde{\lambda}(t-\tau, \sigma) u(t-\tau+\sigma) \mathrm{d} \sigma \\
& \left.+\tilde{k}^{\prime} r_{0}\right] \\
e_{1}(t)= & h_{\mathrm{m}}^{T} e(t) .
\end{aligned}
$$

which can also be written as

$$
\begin{aligned}
\dot{e}(t)= & A_{\mathrm{m}} e(t)+b_{\mathrm{m}}\left[\tilde{\theta}^{\prime} T(t-\tau) \Omega(t-\tau)\right. \\
& \left.+\int_{-\tau}^{0} \tilde{\lambda}(t-\tau, \sigma) u(t-\tau+\sigma) \mathrm{d} \sigma\right] \\
e_{1}(t)= & h_{\mathrm{m}}^{T} e(t) .
\end{aligned}
$$


where, $\tilde{\theta}^{\prime}=\left[\begin{array}{ccc}\tilde{\alpha}_{1} & \tilde{\alpha}_{2} & \tilde{k}^{\prime}\end{array}\right]^{T}$. Equations (37) and (34) are exactly the same equations written using different variables, meaning that the definition of the new variable does not alter the equilibrium position of the differential equation. In addition, (37) is in the same form as in the case of disturbance free system (24), and hence the stability proof follows along the same lines and $\lim _{t \rightarrow \infty} e_{1}(t)=0$. Therefore, the system is stable, the disturbance is rejected and the plant output follows the reference model output asymptotically.

To conclude, disturbance rejection is achieved by eliminating the disturbance term in the error equation and this is done by introducing a new variable defined by shifting the feedforward controller term $k$ by a constant.

\section{Acknowledgment}

This work was supported through the Ford-MIT Alliance Initiative. The authors would like to acknowledge Dr. Davor Hrovat of Ford Motor Company for his support and encouragement during this project. The authors also wish to acknowledge Steve Magner and John Michelini of Ford Motor Company for their help and valuable discussions.

\section{References}

Arsie, I., Pianese, C., Rizzo, G., Cioffi, V., Mar. 2003. An adaptive estimator of fuel film dynamics in the intake port of a spark ignition engine. Control Eng. Practice 11 (3), 303-309.

Ault, B., Jones, V. K., Powell, J. D., Franklin, G. F., 1994. Adaptive air-fuel ratio control of a spark-ignition engine. SAE Paper (940373). 
Chang, C.-F., Fekete, N. P., Amstutz, A., Powell, J. D., Mar. 1995. Airfuel ratio control in spark-ignition engines using estimation theory. IEEE Transactions on Control Systems Technology 3 (1).

Chang, C. F., Fekete, N. P., Powell, J. D., 1993. Engine air-fuel ratio control using an event-based observer. In: Proc. of SAE. No. 930766.

Choi, S. B., Hedrick, J. K., May 1998. An observer-based controller design method for improving air/fuel characteristics of spark ignition engines. IEEE Transactions on Control Systems Technology 6 (3), 325-334.

Engelborghs, K., Dambrine, M., Roose, D., Feb. 2001. Limitations of a class of stabilization methods for delay systems. IEEE Trans. Automatic Control $46(2)$.

Guzzella, L., 1995. Models and model-based control of IC-engines A nonlinear approach. SAE Paper (950844).

Guzzella, L., Onder, C. H., 2004. Introduction to Modeling and Control of Internal Combustion Engine Systems, 1st Edition. Springer.

Guzzella, L., Simons, M., Geering, H. P., Aug. 1997. Feedback linearizing air/fuel-ratio controller. Control Eng. Practice 5 (8), 1101-1105.

Ichikawa, K., 1985. Frequency-domain pole assignement and exact modelmatching for delay systems. International Journal of Control 41, 10151024 .

Jones, V. K., Ault, B. A., Franklin, G. F., Powell, J. D., Mar. 1995. Identifica- 
tion and air-fuel ratio control of a spark ignition engine. IEEE Transactions on Control Systems Technology 3 (1).

Karason, S. P., Annaswamy, A. M., Nov. 1994. Adaptive control in the presence of input constraints. IEEE Trans. Automatic Control 39 (11), 23252330 .

Majhi, S., Atherton, D. P., Nov. 2000. Obtaining controller parameters for a new Smith predictor using autotuning. Automatica 36 (11), 1651-1658.

Manitius, A. Z., Olbrot, A. W., 1979. Finite spectrum assignement problem for systems with delays. IEEE Transactions on Automatic Control 24 (4).

Mianzo, L., Peng, H., Haskara, I., 2001. Transient air-fuel ratio $H_{\infty}$ preview control of a drive-by-wire internal combustion engine. In: Proc. Amer. Control Conf. pp. 2867-2871.

Muske, K. R., Jones, C. P. J., 2006. A model-based si engine air fuel ratio controller. In: Proc. Amer. Control Conf. pp. 3284-3289.

Nakagawa, S., Katogi, K., Oosuga, M., 2002. A new air-fuel ratio feedback control for ulev/sulev standard. SAE Paper (2002-01-0194).

Narendra, K. S., Annaswamy, A. M., 2005. Stable adaptive systems. Dover Publications, New York.

Niculescu, S.-I., Annaswamy, A. M., 2003. An adaptive smith-controller for time-delay systems with relative degree $n^{*} \leqslant 2$. Systems and Control Letters $49,347-358$. 
Ohata, A., Ohashi, M., Nasu, M., Inoue, T., 1995. Crank-angle domain modeling and control for idle speed. SAE Paper (950075).

Onder, C. H., Geering, H. P., 1993. Model-based multivariable speed and air-to-fuel ratio control of an si engine. SAE Paper (930859).

Ortega, R., Lozano, R., 1988. Globally stable adaptive controller for systems with delay. International Journal of Control 47 (1), 17-23.

Pieper, J. K., Mehrotra, R., June 1999. Air/fuel ratio control using sliding mode methods. In: Proc. Amer. Control Conf. San Diego, CA, pp. 10271031.

Powell, J. D., Fekete, N. P., Chang, C. F., Oct. 1998. Observer-based air-fuel ratio control. IEEE Control Systems Magazine 18 (5), 72-83.

Rupp, D., February 2009. Model-based adaptive air/fuel ratio control for an automotive gasoline engine. Ph.D. thesis, ETH Zurich.

Rupp, D., Onder, C., Guzzella, L., 2008. Iterative adaptive air/fuel ratio control. In: Proc. Advances in Automotive Control. Seascape Resort, USA.

Shelef, M., McCabe, R. W., Sep. 2000. Twenty-five years after introduction of automotive catalysts: what next? Catalysis Today 62 (1), 35-50.

Smith, O. J., 1959. A controller to overcome dead time. ISA Journal 6.

Souder, J. S., Hedrick, J. K., Apr. 2004. Adaptive sliding mode control of air-fuel ratio in internal combustion engines. Int. J. Robust Nonlin. Cont. $14(6), 525-541$. 
Stefanopoulou, A. G., Grizzle, J. W., Freudenberg, J. S., 1994. Engine air-fuel ratio and torque control using secondary throttles. In: Proc. of Conference on Decision and Control. pp. 2748-2753.

Tunestal, P., Hedrick, J. K., Mar. 2003. Cylinder air/fuel ratio estimation using net heat release data. Control Eng. Practice 11 (3), 311-318.

Turin, R., Geering, H., 1995. Model-reference adaptive A/F ratio control in an SI engine based on Kalman-Filtering techniques. In: Proc. Amer. Control Conf. pp. 4082-4090.

van Nieuwstadt, M. J., Kolmanovsky, I. V., Moraal, P. E., Stefanopoulou, A., Jankovic, M., Jun. 2000. EGR-VGT control schemes: experimental comparison for a high-speed diesel engine. IEEE Control Systems Magazine $20(3), 63-79$.

Vigild, C. W., Andersen, K. P. H., Hendricks, E., Struwe, M., 1999. Towards robust H-infinity control of an SI engine's air/fuel ratio. SAE Paper (199901-0854).

Won, M., Choi, S. B., Hedrick, J. K., Sep. 1998. Air-to-fuel ratio control of spark ignition engines using gaussian network sliding control. IEEE Transactions on Control Systems Technology 6 (5), 678-687.

Yildiz, Y., Annaswamy, A., Kolmanovsky, I., Yanakiev, D., 2009a. Adaptive posicast controller for time-delay systems with relative degree $n^{*} \leqslant 2$. under review http://web.mit.edu/aaclab/publications/index.html. 
Yildiz, Y., Annaswamy, A., Yanakiev, D., Kolmanovsky, I., July 2007. Adaptive idle speed control for internal combustion engines. In: Proc. Amer. Control Conf. New York City, pp. 3700-3705.

Yildiz, Y., Annaswamy, A., Yanakiev, D., Kolmanovsky, I., June 2008a. Adaptive air fuel ratio control for internal combustion engines. In: Proc. Amer. Control Conf. Seattle, Washington, pp. 2058-2063.

Yildiz, Y., Annaswamy, A., Yanakiev, D., Kolmanovsky, I., Oct. 2008b. Automotive powertrain control problems involving time delay: An adaptive control approach. In: Proc. of ASME Dynamic Systems and Control Conference. Ann Arbor, Michigan.

Yildiz, Y., Annaswamy, A., Yanakiev, D., Kolmanovsky, I., 2009b. Spark ignition engine idle speed control: An adaptive control approach. under review http://web.mit.edu/aaclab/publications/index.html.

Zhai, Y.-J., Yu, D.-L., in press. Neural network model-based automotive engine air/fuel ratio control and robustness evaluation. Engineering Applications of Artificial Intelligence.

Zhang, F., Grigoriadis, K., Franchek, M., Makki, I., 2007. Linear parameter varying lean burn air-fuel ratio control for a spark ignition engine. Journal of Dynamic Systems, Measurement and Control 129, 404-414. 